\title{
Adaptive Subspace Predictive Control with Time-varying Forgetting Factor
}

\author{
Li Zhang $^{1} \quad$ Shan-Zhi Xu ${ }^{2} \quad$ Hong-Tao Zhao ${ }^{3}$ \\ ${ }^{1}$ School of Mechanical, Electrical and Information Engineering, Shandong University at Weihai, Weihai 264209, China \\ ${ }^{2}$ Electrical Technology Department of Taizhong Group Yuci Hydraulics Industry Co., Ltd, Jinzhong 030600, China \\ ${ }^{3}$ Power Generation Plant of Cpi Fushun Thermo Electrical Power Co., Ltd, Fushun 030600, China
}

\begin{abstract}
Aiming at the time-varying characteristics of industrial process, this paper introduces an adaptive subspace predictive control (ASPC) strategy with time-varying forgetting factor based on the original subspace predictive control algorithm (SPC). The new method uses model matching error to calculate the variable forgetting factor, and applies it to constructing Hankel data matrix. This makes the data represent the changes of system information better. For eliminating the steady state error, the derivation of the incremental control is made. Simulation results on a rotary kiln show that this control strategy has achieved a good control effect.
\end{abstract}

Keywords: Subspace predictive control, time-varying forgetting factor, model matching error, adaptive, rotary kiln.

\section{Introduction}

Model predictive control (MPC), which is a control algorithm able to handle constrained complex systems, has been widely used in process industry. However, MPC is facing a practical problem that is how to deal with uncertainty of the dynamic characteristics model, so that the system can guarantee good performance. Therefore, it is one of the hottest spots in the MPC field that how to establish an accurate prediction model when the system exists uncertainty ${ }^{[1-3]}$.

Subspace method identifies a system by input and output data, and its algorithm uses some simple and reliable linear algebra methods. Its good capacity to deal with multivariate system is very suitable for use as a predictive control model ${ }^{[4,5]}$.

Subsequently, the predictive control based on subspace identification method appeared in different studies. Some of them used subspace predictor matrix, and others utilized the subspace identification to obtain the state-space model as a predictive model. Kadali et al. ${ }^{[1]}$ proposed a design predictive control method by using subspace predictor directly, and the typical characteristics of predictive control were included. In recent years, subspace predictive control (SPC) has acquired more research results ${ }^{[6,7]}$. Huang and Kadali ${ }^{[6]}$ designed the SPC in the general predictive control (GPC). However, the forgetting factor mechanism was not considered and this affected the tracking speed of the algorithm parameters. In [8] a novel recursive predictor-based subspace identification method was presented to identify linear time-invariant systems with multiinputs and multi-outputs. The method was implemented in real-time and was able to operate in open loop or closed loop. Alenanya and Shang ${ }^{[9]}$ developed a recursive version of the constrained least squares (CLS) subspace algorithm. The advantage of this approach is that the CLS subspace algorithm is a linear regression problem and hence its recursive algorithm can be obtained without updating neither the

Manuscript received May 8, 2013; revised July 18, 2013 observability matrix, SVD, nor QR decompositions ${ }^{[9]}$. In recent years many SPC methods have been studied ${ }^{[10-12]}$.

However, the SPC method used in actual industrial process is rare. In this paper, a subspace predictive control algorithm is deduced. Considering the existence of timevarying characteristics in the lime calcination process, a variable forgetting factor recursive strategy, adaptive subspace predictive control (ASPC) strategy is proposed. This strategy uses the matching error of the model to calculate the time-varying forgetting factor, which is in the construction of the data matrix. Thus the data will follow the system information better, and the optimal control will be obtained. Finally, the incremental control law is employed to eliminate the steady state error.

\section{Problem statement}

We consider the following uncertain discrete-time (LTI) system:

$$
\left\{\begin{array}{l}
x(k+1)=A x(k)+B u(k)+K e(k) \\
y(k)=C x(k)+D u(k)+e(k)
\end{array}\right.
$$

where $k$ represents the $k$-th sampling time, $K$ is the Kalman gain of the stable state. $u(k) \in \mathbf{R}^{n_{u}}, x(k) \in \mathbf{R}^{n_{x}}, y(k) \in$ $\mathbf{R}^{n_{y}}$ are the input, the unmeasurable state, and the output, respectively. $\{e(k)\}$ is zero-mean Gaussian white noise, and $A, B, C, D$ are the corresponding dimension matrices.

For system (1), the control system is shown as Fig. 1.

And the input and output data sets are $\left(\left[\begin{array}{c}u(1) \\ u(2) \\ \vdots \\ u(n)\end{array}\right],\left[\begin{array}{c}y(1) \\ y(2) \\ \vdots \\ y(n)\end{array}\right]\right)$ with measuring length $N$.

Here the predictive method is adopted, which recursively identifies $L_{w}$ and $L_{U}$ online. This avoids the molding process. The process can be explained as looking for the optimal predictive value of a future output based on the past 
input/output matrix $W_{p}$ and future input matrix $U_{f}$. This can be expressed as a linear predictor:

$$
\hat{Y}_{f}=L_{\omega} W_{p}+L_{u} U_{f}
$$

The prediction of minimum-square of $Y_{f}$ is $\hat{Y}_{f}$, which can be obtained through solving the following minimum variance problem:

$$
\min _{L_{\omega}, L_{u}}=\left\|Y_{f}-\left(\begin{array}{cc}
L_{\omega} & L_{u}
\end{array}\right)\left(\begin{array}{c}
W_{p} \\
U_{f}
\end{array}\right)\right\|_{F}^{2} .
$$

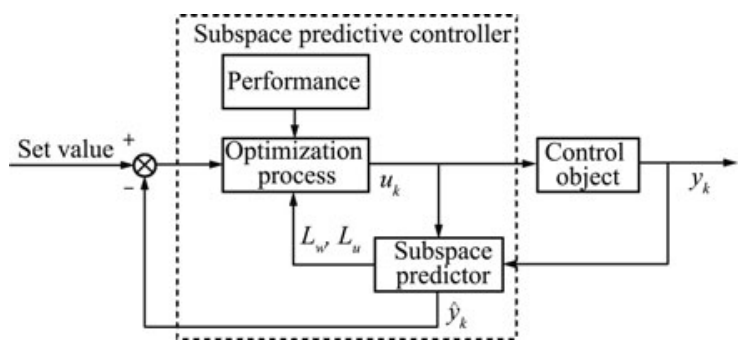

Fig. 1 The SPC system

\section{ASPC with time-varying forgetting factor}

$L_{\omega}$ and $L_{u}$ play an important role in solving the predictive control law. Therefore, the foremost problem is to obtain accurate information of these two matrices. In each iteration, the model is updated by adding new data ${ }^{[13,14]}$. Furthermore, a novel recursive subspace identification algorithm based on forgetting factors is proposed.

\subsection{Hankel matrices with time-varying forgetting factor}

In this paper, we calculate the time-varying forgetting factor using model matching error. First, we take the current model error and its integration as a measure of the model matching degree. Define the model matching degree as

$$
\Delta(k)=\alpha e^{2}(k)+\beta \sum_{j=1}^{L} \theta^{j} e^{2}(k-j)
$$

where $e(k)=\hat{y}(k)-y(k), \hat{y}(k)$ is the calculated output at $k, y(k)$ is the actual output at $k, \alpha$ is current error weight, $\beta$ is the combination weight of past time, $\theta$ is memory effect parameters of matching error, $L$ is the error length of past time. Then, we define the time-varying forgetting factor $\lambda(k)=\varepsilon / \Delta(k), \varepsilon<\min \Delta(k)$ represents the permissible degree of the model matching error.

When the model-matching error is large, it will significantly accelerate the forgetting of old data with the correction model by new data. When the matching error is close to the permissible degree, the model is only corrected slightly ${ }^{[15]}$.

We construct the new Hankel matrix $\tilde{U}_{p}^{\lambda(k)}$ using the new input $u(k)$ and $y(k)$ :

$\tilde{U}_{p}^{\lambda(k)}=\left[\begin{array}{ccc}\Pi(k)^{k} u(0) & \cdots & \Pi(k)^{i-1} u(k-i+1) \\ \Pi(k)^{k-1} u(1) & \cdots & \Pi(k)^{i-2} u(k-i+2) \\ \vdots & \vdots & \vdots \\ \Pi(k)^{k-i+1} u(i-1) & \cdots & u(k)\end{array}\right]$

$$
\tilde{Y}_{p}^{\lambda(k)}=\left[\begin{array}{ccc}
\Pi(k)^{k} y(0) & \cdots & \Pi(k)^{i-1} y(k-i+1) \\
\Pi(k)^{k-1} y(1) & \cdots & \Pi(k)^{i-2} y(k-i+2) \\
\vdots & \vdots & \vdots \\
\Pi(k)^{k-i+1} y(i-1) & \cdots & y(k)
\end{array}\right]
$$

where $\Pi(k)^{i}=\lambda(k) \lambda(k-1), \cdots, \lambda(k-i+1)$, the new Hankel matrix can also be written as $\tilde{Y}_{p}^{\lambda(k)}=$ $\Lambda_{P}(k) Y_{p} \Lambda(k), \tilde{U}_{p}^{\lambda(k)}=\Lambda_{m}(k) U_{p} \Lambda(k)$, where $m$ and $p$ are the corresponding dimensions, $\Lambda_{P}(k), \Lambda_{m}(k)$ and $\Lambda(k)$ are

$$
\begin{gathered}
\Lambda_{p}(k)=\operatorname{diag}\left\{\Pi(k)^{i-1} I_{p}, \cdots, \Pi(k)^{1} I_{P}, I_{P}\right\} \\
\Lambda_{m}(k)=\operatorname{diag}\left\{\Pi(k)^{i-1} I_{m}, \cdots, \Pi(k)^{1} I_{m}, I_{m}\right\} \\
\Lambda(k)=\operatorname{diag}\left\{\Pi(k)^{i-1}, \cdots, \Pi(k)^{1}, 1\right\} .
\end{gathered}
$$

The $\tilde{U}_{f}^{\lambda(k)}$ and $\tilde{Y}_{f}^{\lambda(k)}$ have the same definition.

\subsection{ASPC control law design}

Consider the following objective function, for which if $N_{1}=1$ then

$$
\begin{aligned}
J= & \sum_{j=1}^{N_{2}}\left(r_{k+j}-\hat{y}_{k+j \mid k}\right)^{2}+\sum_{j=1}^{N_{u}} \lambda\left(u_{k+j-1}\right)^{2}= \\
& \left(r_{f}-\hat{y}_{f}\right)^{\mathrm{T}}\left(r_{f}-\hat{y}_{f}\right)+u_{f}^{\mathrm{T}}(\lambda I) u_{f} .
\end{aligned}
$$

Here, the future output range is equal to the prediction horizon, from $k+1$ to $k+N_{2}$. The future increment input range is equal to the control horizon, from $k$ to $k+N_{u}-1$. $r_{f}$ is the reference input. In the state space model, the optimal prediction of future output vector can be represented as the future input and the current state.

$$
\begin{aligned}
& \hat{y}_{f}=\left[\begin{array}{llll}
\hat{y}_{k+1} & \hat{y}_{k+2} & \cdots & \hat{y}_{k+N_{2}}
\end{array}\right]^{\mathrm{T}}= \\
& {\left[\begin{array}{c}
C \\
C A \\
\cdots \\
C A^{N_{2}-1}
\end{array}\right] x_{t}+\left[\begin{array}{cccc}
D & 0 & \cdots & 0 \\
C B & D & \ddots & 0 \\
\vdots & \ddots & \ddots & \vdots \\
C A^{N_{2}-2} B & C A^{N_{2}-3} B & \cdots & D
\end{array}\right] \times} \\
& {\left[\begin{array}{c}
u_{k} \\
u_{k+1} \\
\vdots \\
u_{k+N_{u}-1}
\end{array}\right]=} \\
& \Gamma_{N_{2}} x_{k}+H\left(1: m N_{2}, 1: l N_{u}\right) u_{f}= \\
& L_{w}\left(1: m N_{2},:\right) w_{p}+L_{u}\left(1: m N_{2}, 1: l N_{u}\right) u_{f}
\end{aligned}
$$


where $w_{p}=\left[\begin{array}{lllllll}y_{k-i+1} & \cdots & y_{k-1} & y_{k} & u_{k-i} & \cdots & u_{k-2}\end{array}\right.$ $\left.u_{k-1}\right]^{\mathrm{T}}$, and $u_{f}=\left[\begin{array}{lllll}u_{k} & u_{k+1} & \cdots & u_{k+N_{u}-1}\end{array}\right]^{\mathrm{T}}$. Substituting (11) into the objective function (10), we have

$$
J=\sum_{j=1}^{N_{2}}\left(r_{k+j}-\left(L_{w} w_{p}+L_{u} u_{f}\right)\right)^{2}+\sum_{j=1}^{N_{u}} \lambda\left(u_{k+j-1}\right)^{2}
$$

In finite horizon $\left\{N_{2}, N_{u}\right\}$, we make the derivation of $J$ equal to zero. Then we get the SPC control law:

$$
u_{f}=\left(\lambda I+L_{u}^{\mathrm{T}} L_{u}\right)^{-1} L_{u}^{\mathrm{T}}\left(r_{f}-L_{w} w_{p}\right)
$$

where $w_{p}=W_{p}(:, 1)$ is the control input. The ASPC control method with time-varying forgetting factor is as follows:

Step 1. Construct Hankel matrices $U_{f}, U_{f}, W_{p}=$ $\left[\begin{array}{c}Y_{p} \\ U_{p}\end{array}\right]$ based on the input and output data.

\section{Step 2. Calculate}

$$
\left[\begin{array}{c}
W_{p} \\
U_{f} \\
Y_{f}
\end{array}\right]=\left[\begin{array}{ccc}
R_{11} & 0 & 0 \\
R_{21} & R_{22} & 0 \\
R_{31} & R_{32} & R_{33}
\end{array}\right]\left[\begin{array}{c}
Q_{1}^{\mathrm{T}} \\
Q_{2}^{\mathrm{T}} \\
Q_{3}^{\mathrm{T}}
\end{array}\right]
$$

Step 3. Calculate $L=\left[\begin{array}{ll}L_{w} & L_{u}\end{array}\right]=$ $\left[\begin{array}{ll}R_{31} & R_{32}\end{array}\right]\left[\begin{array}{cc}R_{11} & 0 \\ R_{21} & R_{22}\end{array}\right]^{\dagger}$ and get the predictive controller parameters:

$$
\begin{aligned}
& L_{w}=L(:, 1: N(m+l)) \\
& L_{u}=L(:, N(m+l)+1: \text { end }) .
\end{aligned}
$$

Step 4. Construct the controller input:

$$
\begin{aligned}
& w_{p}=\left[\begin{array}{llllll}
y_{-N+1}^{\mathrm{T}} & \cdots & y_{-1}^{\mathrm{T}} & y_{0}^{\mathrm{T}} & \cdots
\end{array}\right. \\
& \left.\begin{array}{llll}
u_{-N+1}^{\mathrm{T}} & \cdots & u_{-1}^{\mathrm{T}} & u_{0}^{\mathrm{T}}
\end{array}\right] .
\end{aligned}
$$

Step 5. Calculate the predictive control sequence $u_{f}=$ $\left(\lambda I+L_{u}^{\mathrm{T}} L_{u}\right)^{-1} L_{u}^{\mathrm{T}}\left(r_{f}-L_{w} w_{p}\right)$ and carry out the first control $u_{1}$.

Step 6. Measure the system output $y_{1}$, and return to Step 2.

\subsection{Incremental control}

The above control law tracks the reference input trajectory by the control variable $u$, but does not incrementally control $\Delta u$. There is a steady-state error. So it is needed to that use the objective function with increment input as follows:

$$
\begin{aligned}
J= & \sum_{j=1}^{N_{2}}\left(r_{k+j}-\hat{y}_{k+j \mid k}\right)^{2}+\sum_{j=1}^{N_{u}} \lambda\left(\Delta u_{k+j-1}\right)^{2}= \\
& \left(r_{f}-\hat{y}_{f}\right)^{\mathrm{T}}\left(r_{f}-\hat{y}_{f}\right)+\Delta u_{f}^{\mathrm{T}}(\lambda I) \Delta u_{f} .
\end{aligned}
$$

Suppose $e_{k}$ is the integration noise, that is,

$$
e_{k+1}=e_{k}+a_{k}+1
$$

where $a_{k}$ is white noise signal, and $\Delta=1-z^{-1}$ is difference operator $e_{k}=a_{k} / \Delta$. Then, we can get

$$
z_{k+1}=A z_{k}+B \Delta u_{k}+K^{f} a_{k}
$$

$$
\Delta y_{k}=C z_{k}+D \Delta u_{k}+a_{k}
$$

where $z_{k}=x_{k}-x_{k-1}$, so the subspace input and output expression becomes

$$
\Delta Y_{f}=\Gamma_{i} Z_{f}+H_{i} \Delta U_{f}+H_{i}^{s} A_{f}
$$

$$
\Delta \hat{Y}_{f}=\Gamma_{i} z_{k}+H_{i} \Delta u_{f}=L_{w}\left[\begin{array}{c}
\Delta y_{p} \\
\Delta u_{p}
\end{array}\right]+L_{u} \Delta u_{f} .
$$

The $j$ step predictor is

$$
\begin{gathered}
y_{k+j-1}-y_{k-1}=\left(C A^{j-1}+\cdots+C\right) z_{k}+ \\
{\left[\left(C A^{j-1} B+\cdots+D\right) \Delta u_{k}+\cdots+D \Delta u_{k+j-1}\right]+} \\
{\left[a_{k}+a_{k+1}+\cdots+a_{k+j-1}\right] .}
\end{gathered}
$$

Equation (11) becomes

$$
\begin{aligned}
& \hat{Y}_{f}=\left[\begin{array}{llll}
\hat{Y}_{k+1} & \hat{Y}_{k+2} & \cdots & \hat{Y}_{k+N_{2}}
\end{array}\right]^{\mathrm{T}}= \\
& \bar{y}_{k}+\Gamma_{N_{2}}^{\circ} z_{k}+S_{N_{2}, N_{u}} \Delta u_{f}= \\
& \bar{y}_{k}+L_{w}^{\circ}\left(1: N_{2} m,:\right)\left[\begin{array}{c}
\Delta y_{p} \\
\Delta u_{p}
\end{array}\right]+S_{N_{2}, N_{u}} \Delta u_{f}= \\
& F+S_{N_{2}, N_{u}} \Delta u_{f}
\end{aligned}
$$

where $\Gamma_{N_{2}}^{\circ}$ is the improved generalized observation matrix; $S_{N_{2}, N_{u}}$ is the $\left(N_{2} m \times N_{u} l\right)$ dimensional dynamic matrix. And $\bar{y}_{k}=\left[\begin{array}{c}y_{k} \\ y_{k} \\ \vdots \\ y_{k}\end{array}\right], \Gamma_{N_{2}}^{\circ}=\left[\begin{array}{c}C \\ C A+C \\ \vdots \\ C A^{N_{2}-1}+\cdots+C\end{array}\right]$ and $S_{N_{2}, N_{u}}=L_{u}\left(1: N_{2} m, 1: N_{u} l\right)\left[\begin{array}{ccc}I_{l} & \cdots & 0 \\ I_{l} & \cdots & 0 \\ \vdots & \ddots & \vdots \\ I_{l} & \cdots & I_{l}\end{array}\right] . L_{w}^{\circ}$ is constructed by $L_{w}$ :

$$
\begin{aligned}
& L_{w}^{\circ}(m(j-1)+1: m j,:)= \\
& \quad \sum_{i=1}^{j} L_{w}(m(i-1)+1: m j,:)
\end{aligned}
$$

where $1 \leqslant j \leqslant N_{2}, F$ is zero input response:

$$
F=y_{k}+L_{w}^{\circ}\left(1: N_{2} m,:\right)\left[\begin{array}{c}
\Delta y_{p} \\
\Delta u_{p}
\end{array}\right] \text {. }
$$

Substituting the prediction equation (24) into the objective function (17) yields

$$
\begin{aligned}
J= & \left(r_{f}-F-S_{N_{2}, N_{u}} \Delta u_{f}\right)^{\mathrm{T}}\left(r_{f}-F-S_{N_{2}, N_{u}} \Delta u_{f}\right) y_{k}+ \\
& \Delta u_{f}^{\mathrm{T}}(\lambda I) \Delta u_{f} .
\end{aligned}
$$

Get the incremental control by derivation of $J$ :

$$
\Delta u_{f}=\left(S_{N_{2}, N_{u}}^{\mathrm{T}} S_{N_{2}, N_{u}}+\lambda I\right)^{-1} S_{N_{2}, N_{u}}^{\mathrm{T}}\left(r_{f}-F\right) .
$$


Because only $\Delta u_{f}(1)$ is executed and is recalculated each time interval, in the time interval we can only calculate $\Delta u_{k}=\Delta u_{f}(1)=m_{l}\left(r_{f}-F\right)$, where $m_{l}$ is the front row of the matrix $\left(S_{N_{2}, N_{u}}^{\mathrm{T}} S_{N_{2}, N_{u}}+\lambda I\right)^{-1} S_{N_{2}, N_{u}}^{\mathrm{T}}$. So $u_{k}$ is

$$
\begin{aligned}
u_{k}= & u_{k-1}+\Delta u_{k}= \\
& u_{k-1} m_{l}\left(r_{f}-\left(\bar{y}_{k}+L_{w}^{\circ}\left(1: N_{2} m,:\right)\left[\begin{array}{c}
\Delta y_{p} \\
\Delta u_{p}
\end{array}\right]\right)\right) .
\end{aligned}
$$

\section{Simulation study}

\subsection{Rotary kiln model}

The rotary kiln is the key equipment in metallurgical industry. Product quality, energy consumption, production safety and cost are directly related to the running state of the rotary kiln. Calcination temperature in the rotary kiln is an important process parameter that plays a decisive role to ensure product quality. The lime kiln under study is $600 \mathrm{t} / \mathrm{d}, 4.0 \mathrm{~m} \times 60 \mathrm{~m}$. The main factor that affects the quality of lime rotary kiln is calcining zone temperature, the ideal temperature range is between $1260-1340{ }^{\circ} \mathrm{C}$. In this paper, the calcining zone temperature is taken as the controlled object, and the gas flow is taken as the control.

The calcining zone state-space model of the rotary kiln is built by using PO-Moesp subspace method:

$$
\begin{aligned}
A & =\left[\begin{array}{ccc}
-0.4254 & 0.3016 & -0.0018 \\
-0.3029 & 0.1939 & 3.4207 \mathrm{E}-005 \\
-0.0001 & -8.0371 \mathrm{E}-006 & -0.6812
\end{array}\right] \\
B & =\left[\begin{array}{c}
-0.0641 \\
-0.0045 \\
2.6873 \mathrm{E}-009
\end{array}\right] \\
C & =[-3.5981-0.60216 .3719 \mathrm{E}-012] \\
D & =3.5067 \mathrm{E}-017 .
\end{aligned}
$$

Its corresponding controlled auto regressive integrated moving average (CARIMA) model is

$$
G\left(z^{-1}\right)=\frac{0.02283 z^{-1}-0.04466 z^{-2}+0.02179 z^{-3}}{1-2.911 z^{-1}+2.824 z^{-2}-0.9128 z^{-3}} .
$$

\subsection{Simulation results}

Set the target temperature to be 1200 , the simulation time is 500 minutes. According to the demands from the industrial scene, set the constraints range of $u$ to be 0 to $11000 \mathrm{~m}^{3} / h$ and $\Delta u$ to be -1000 to $1000 \mathrm{~m}^{3} / h$. The control effect is shown as Fig. 2. The result of simulation indicates that the ASPC method has good control effect and eliminates the steady-state error. Then, we compare this model with GPC in Fig. 3. It can be seen that both them can eliminate the steady-state error, but the subspace adaptive predictive control response speed is faster than the GPC.
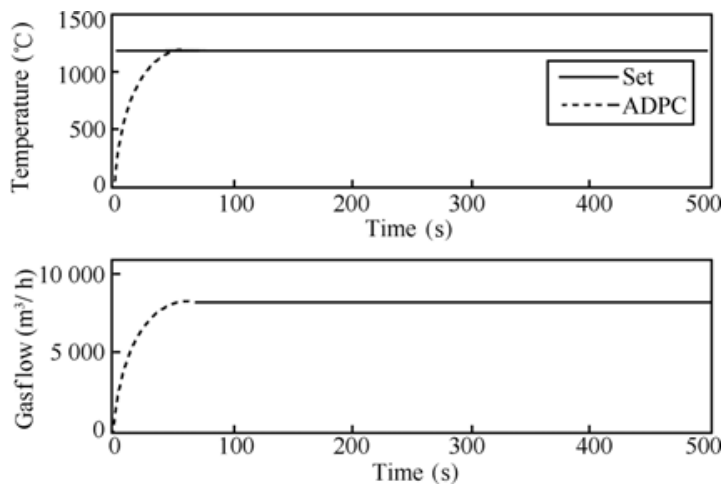

Fig. 2 The diagram of ASPC control effect

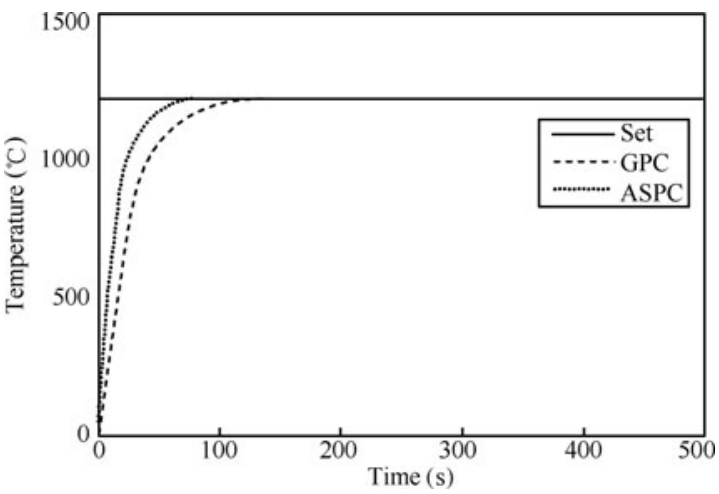

Fig. 3 The comparison diagram of ASPC and GPC

Suppose the set temperature changes from $1200{ }^{\circ} \mathrm{C}$ to $1300{ }^{\circ} \mathrm{C}$ at time $200 \mathrm{~min}$, and back to $1200^{\circ} \mathrm{C}$ at $350 \mathrm{~min}$. The control effect is shown in Fig. 4. As can be seen, the subspace has a better tracking ability.

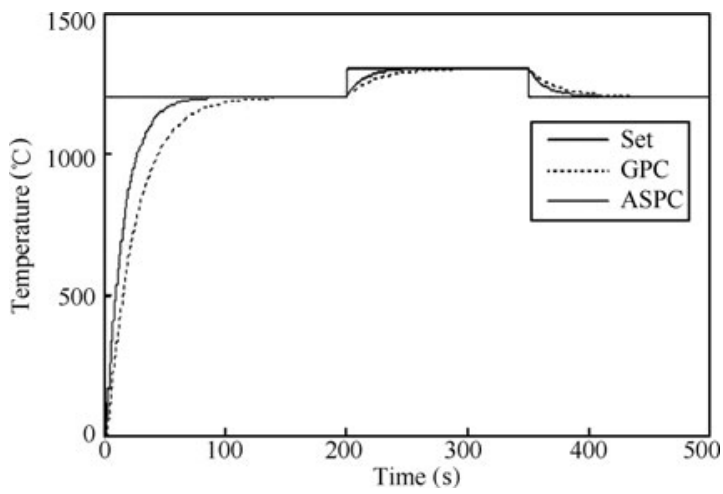

Fig. 4 The comparison diagram of set temperature changed

In Fig. 5, the system changes at time $300 \mathrm{~min}$. The $B\left(z^{-1}\right)$ changed into:

$$
B\left(z^{-1}\right)=0.02283-0.54466 z^{-1}+0.02179 z^{-2} .
$$

When the model mismatch, adaptive subspace predictive control can achieve better results. Fig. 6 study the changes of model matching errors $\Delta(k)$, when $\lambda$ is $1,0.9$ and variable, respectively. 


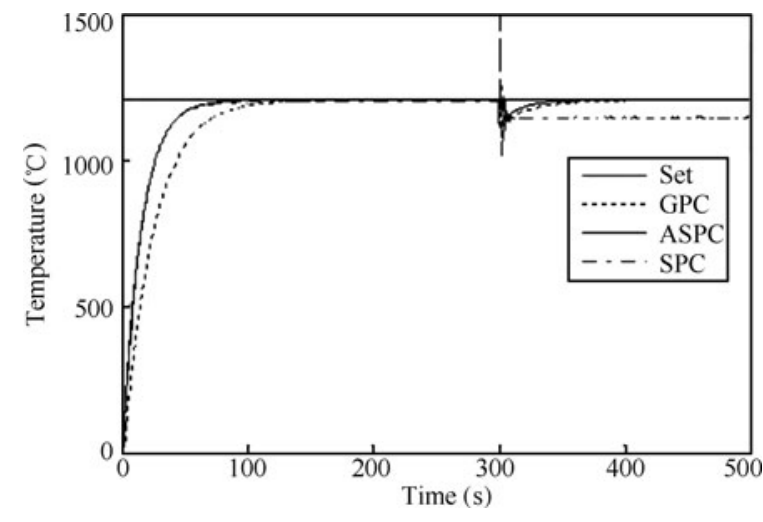

Fig. 5 The comparison diagram of controlled object changed

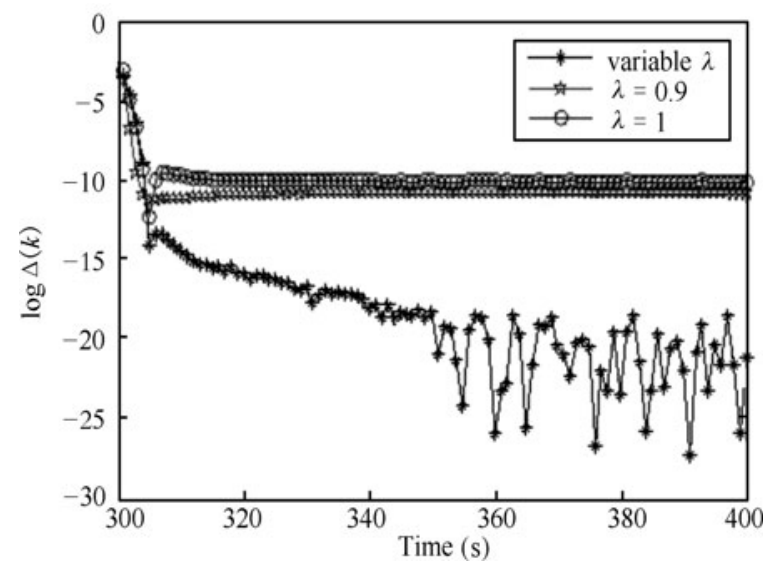

Fig. 6 The comparison diagram of model matching errors

\section{Conclusions}

Focusing on the problem of algorithm performance degradation which is caused by the model mismatch, we propose a variable forgetting factor subspace predictive control strategy. The time-varying forgetting factor was calculated based on the model-matching error. After designing the control law, the incremental control law is derived, and this eliminates the steady state error. Finally, the simulation was carried out, which took the rotary kiln as the control object.

\section{References}

[1] R. Kadali, B. Huang, A. Rossiter. A data driven subspace approach to predictive controller design. Control Engineering Practice, vol. 11, no. 3, pp. 261-278, 2003.

[2] N. A. Mardi, L. P. Wang. Subspace-based model predictive control of time-varying systems. In Proceedings of Joint 48th IEEE Conference on Decision and Control and 28th Chinese Control Conference, IEEE, Piscataway, USA, pp. 4005-4010, 2009.

[3] Y. K. Ho, F. S. Mjalli. Generalized predictive control with dual adaptation. Chemical Engineering Science, vol. 84, pp. 479-493, 2012.

[4] H. Ghasemi, C. A. Canizares, A. Moshref. Oscillatory stability limit prediction using stochastic subspace identification. IEEE Transactions on Power Systems, vol.21, no. 2, pp. 736-745, 2006.

[5] P. Trnka, V. Havlena. Subspace identification as multi-step predictions optimization. In Proceedings of the IASTED International Conference on Modelling, Czech Republic, pp. 223-228, 2005.
[6] B. Huang, R. Kadali. Dynamic Modeling, Predictive Control and Performance Monitoring: A Data-driven Subspace Approach, Springer, 2008.

[7] L. Sun, X. M. Jin. Model-predictive-control based on subspace identification and its application. Control Theory \& Applications, vol. 26, no. 3, pp.313-315, 2009. (in Chinese)

[8] I. Houtzager, J. W. van Wingerden, M. Verhaegen. Recursive predictor-based subspace identification with application to the real-time closed-loop tracking of flutter. IEEE Transactions on Control Systems Technology, vol. 20, no. 4, pp. 934-949, 2012.

[9] A. Alenany, H. Shang. Recursive subspace identification with prior information using the constrained least squares approach. Computers and Chemical Engineering, vol. 54, pp. 174-180, 2013.

[10] X. S. Luo, G. Q. Zhou, T. Zou. State space model predictive control based on subspace identification. Computer Engineering and Applications, vol.48, no. 3, pp. 234-237, 2012.

[11] Z. Yu, Z. J. Sun, S. J. Qin, T. Y. Chai. Non-stationary Kalman filter parametrization of subspace models with applications to MPC. In Proceedings of the American Control Conference, IEEE, Montreal, QC, USA, pp. 4813-4818, 2012.

[12] J. J. Ma, Z. Q. Zheng, D. W. Hu. Subspace predictive dynamic control allocation for overactuated system with actuator dynamics. Acta Automatica Sinica, vol. 36, no. 1, pp. 130-138, 2010.

[13] Y. Hua, N. Li, S. Y. Li. A data-driven bilinear predictive controller design based on subspace method. Asian Journal of Control, vol. 13, no. 2, pp. 345-349, 2011.

[14] H. Yang, S. Y. Li. A novel recursive MOESP subspace identification algorithm based on forgetting factor. Journal of Control Theory and Applications, vol. 26, no. 1, pp. 69-72, 2009. (in Chinese)

[15] G. Luders, K. S. Narendra. An new canonical form for an adaptive observer. IEEE Transactions on Automatic Control, vol. 18, no. 2, pp. 117-119, 1974.

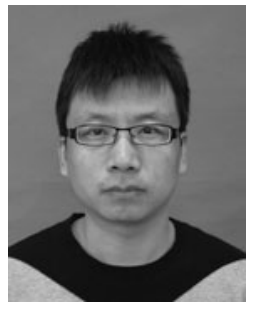

Li Zhang received his B.Sc. and M. Sc. degrees in control science from Liaoning Technical University, Fuxin, China in 2003 and 2007, respectively, and his Ph. D. degree in control science from Northeastern University, Shenyang, China in 2012. He is currently a lecture in School of Mechanical, Electrical and Information Engineering, Shandong University at Weihai, China.

His research interests include model predictive control and industrial process.

E-mail: zhangliwh@sdu.edu.cn (Corresponding author)

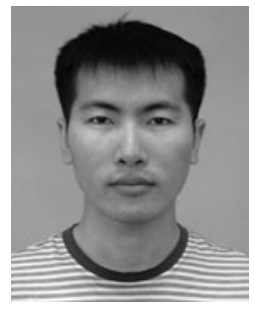

Shan-Zhi Xu received his B.Sc. and M. Sc. degrees in control science from the Northeastern University, China in 2004 and 2011, respectively.

His research interests include predictive controller design and industrial application.

E-mail: xushanzhi@163.com

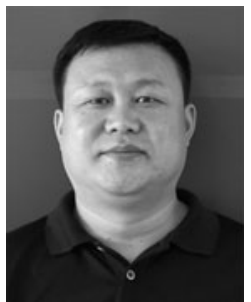

Hong-Tao Zhao received his B. Sc. degree in thermal engineering from Northeast Dianli University, China in 2003.

His research interests include predictive controller design and thermal power generation application.

E-mail: Zhaohongtao@zdtfsrd.com 